\title{
Incidencia de reacciones adversas a medicamentos en pacientes que inician o cambian terapia anti-retroviral
}

\author{
Fernando Bernal, Patricia Vásquez, Carolina Giadalah, Lorena Rodríguez y Alejandra Villagrán
}

\section{Incidence of adverse drug reactions in patients initiating or changing antiretroviral therapy}

Background: In the Chilean AIDS Cohort, the $15.9 \%$ of the patients change the first scheme of HAART because of toxicity, which is also the cause of $47 \%$ suspensions of treatment occurred in the first months of therapy. Aim: To analyze the incidence and causality of adverse drug reactions in patients initiating or changing antiretroviral therapy. Materials and Methods: 92 patients who started or changed antiretroviral treatment in a period from May to July 2011 were followed to detect adverse drug reactions. Results: 75\% (n: 69) of patients had at least one adverse event. From a total of 76 adverse drug reactions detected the most frequent ones were diarrhea $(21.1 \%, \mathrm{n}: 16)$ and hyperbilirubinemia ( $19.7 \% \mathrm{n}: 15) .85 .9 \%$ of the adverse events where grade depending on its seriousness, $34.1 \%$ had grade 1 toxicity, $32.9 \%$ grade $2,9.4 \%$ grade 3 and only $3.5 \%$ grade 4 . Only 22 patients $(31.8 \%)$ required a treatment change. The most frequent causes for changing treatment were because of anaemia by AZT ( $45.4 \%, \mathrm{n}$ : $10)$ and diarrhea associated with the use of LPV/r (27.3\%, n: 6). Conclusion: Knowing the real incidence rate of adverse drug reactions may help the health team giving proper management and monitoring of these reactions in the patient, in order to avoid an early change of HAART. Defining a multidisciplinary team which includes a pharmacist is essential for proper pharmacosurveillance.

Key words: Pharmacosurveillance, adverse drug reactions, antiretroviral therapy, toxicity, HIV Infections.

Palabras clave: Farmacovigilancia, reacción adversa a medicamentos, terapia anti-retroviral, toxicidad, Infección por VIH.

\section{Introducción}

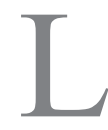

a adherencia al tratamiento anti-retroviral (TARV) ha sido establecida como un pilar fundamental en la reducción de la morbi-mortalidad de la infección por VIH/SIDA, cambiando la historia natural de ésta. Sin embargo, su eficacia terapéutica no está exenta de problemas, ya que se han reportado efectos adversos para todos los anti-retrovirales disponibles, constituyendo la principal causa de falta de adherencia al tratamiento. Actualmente se señala que $96 \%$ de los pacientes que reciben TARV presentan, como mínimo, una situación clínica o un medicamento que podría generar problemas de inseguridad o inefectividad de la terapia. Por tanto, la identificación, prevención y manejo de las reacciones adversas e interacciones medicamentosas se considera un aspecto de gran trascendencia en la consecución de los objetivos terapéuticos en la farmacoterapia de estos pacientes. En Chile, 15,9\% de los pacientes requiere cambio de su primer esquema de TARV por toxicidad, siendo además la causa de $47 \%$ de las suspensiones de tratamiento ocurrida en los primeros meses de terapia. Las reacciones adversas a medicamentos (RAM) causantes de suspensión más frecuentemente informadas por la cohorte Chilena de SIDA son: toxicidad gastrointestinal, hematológica y dermatológica ${ }^{1}$.

\section{Objetivos}

- Analizar la incidencia y causalidad de las reacciones adversas a medicamentos en pacientes que inician o cambian terapia anti-retroviral.

- Graduar la toxicidad de RAM y tiempo de aparición.

- Describir el manejo local de las toxicidades por antiretrovirales.

\section{Metodología}

Se realizó un seguimiento para la detección de RAM, en el control adherencia a 92 pacientes que iniciaron $\mathrm{o}$ cambiaron TARV y cumplieron criterios de inclusión, en un período comprendido entre mayo y julio del año 2011. El programa de adherencia del Hospital San Juan de Dios consiste en un control semanal durante el primer mes tratamiento, donde el inicio de TARV y primer control está a cargo del Químico Farmacéutico del policlínico y posteriormente con Matrona, Psicóloga y Médico, respectivamente. Dado lo anterior y por tratarse de un manejo
Hospital San Juan de Dios, Santiago, Chile.

Lugar de realización:

CDT-Hospital San Juan de Dios, Policlínico de Infectología, Santiago.

Conflicto de interés: ninguno Fuente financiamiento: ninguna

Recibido: 16 de agosto de 2012 Aceptado: 22 de julio de 2013

Correspondencia a: Fernando Bernal Ortiz fernando.bortiz@gmail.com 
habitual de los pacientes, no se requirió su presentación al Comité de Ética institucional.

Durante estos controles cada RAM fue registrada y notificada al Centro Nacional de Información de Medicamentos y Farmacovigilancia (CENIMEF) y graduada según tabla entregada por el MINSAL en su guía clínica VIH/SIDA del año 2009 (Anexo 1).

Para la detección de alteraciones de laboratorio se solicitaron exámenes metabólicos, previo al control médico. Se consideraron todas las RAM independientes de que fueran un evento adverso esperable.

Tabla 1. Características de la población en estudio (n: 92). Reacciones adversas a terapia anti-retroviral. Hospital San Juan de Dios, Chile. 2011

\begin{tabular}{|c|c|c|}
\hline Variables & $\mathbf{n}$ & $\%$ \\
\hline Virgen a tratamiento & 54 & 59,7 \\
\hline Experimentado & 38 & 41,3 \\
\hline \multicolumn{3}{|l|}{ Género } \\
\hline Masculino & 71 & 77,2 \\
\hline Femenino & 21 & 22,8 \\
\hline \multicolumn{3}{|l|}{ Edad (años) } \\
\hline$<20$ & 2 & 2,2 \\
\hline $20-29$ & 23 & 25,0 \\
\hline $30-39$ & 32 & 34,8 \\
\hline $40-49$ & 24 & 26,1 \\
\hline$\geq 50$ & 11 & 11,9 \\
\hline \multicolumn{3}{|l|}{ TARV } \\
\hline 2 INTR +1 INNTR & 41 & 44,6 \\
\hline $2 I N T R+1 I P$ & 48 & 51,2 \\
\hline${ }^{* * \text { Rescate }}$ & 3 & 4,2 \\
\hline
\end{tabular}

Tabla 2. Reacciones adversas pesquisadas ( $\mathrm{n}$ : 76). Estudio de reacciones adversas a terapia anti-retroviral. Hospital San Juan de Dios, Chile. 2011

\begin{tabular}{|c|c|c|c|c|}
\hline RAM & Causal & $\begin{array}{c}n \text { pacientes } \\
\text { expuestos a causal }\end{array}$ & $\begin{array}{l}\text { n pacientes } \\
\text { con RAM }\end{array}$ & $\begin{array}{l}\% \text { del total } \\
\text { de RAM }\end{array}$ \\
\hline Intolerancia gastro-intestinal (diarrea) & $\mathrm{LPV} / \mathrm{r}$ & 29 & 16 & 21,1 \\
\hline Hiperbilirrubinemia indirecta & ATV/r & 18 & 15 & 19,7 \\
\hline $\begin{array}{l}\text { Intolerancia (cefaleas, náuseas, vómitos) } \\
\text { Anemia } \\
\text { Neuropatía periférica }\end{array}$ & $\mathrm{AZT} / 3 \mathrm{TC}$ & 78 & $\begin{array}{r}10 \\
10 \\
2\end{array}$ & $\begin{array}{r}13,2 \\
13,2 \\
2,6\end{array}$ \\
\hline $\begin{array}{l}\text { Alteración SNC } \\
\text { Rash cutáneo } \\
\text { Rash cutáneo } \\
\text { Rash cutáneo }\end{array}$ & $\begin{array}{l}\text { NVP } \\
\text { ETR }\end{array}$ & $\begin{array}{l}7 \\
1\end{array}$ & $\begin{array}{r}10 \\
2 \\
1 \\
1\end{array}$ & $\begin{array}{r}13,2 \\
2,6 \\
1,3 \\
1,3\end{array}$ \\
\hline $\begin{array}{l}\text { Hipercolesterolemia } \\
\text { Hipertrigliceridemia } \\
\text { Hepatotoxicidad }\end{array}$ & TARV & 92 & $\begin{array}{l}1 \\
6 \\
2\end{array}$ & $\begin{array}{l}1,3 \\
7,9 \\
2,6\end{array}$ \\
\hline
\end{tabular}

RAM: reacción adversa a medicamentos; LPV/r: lopinavir/ritonavir; ATV/r: atazanavir/ritonavir; AZT/3TC: zidovudina/lamivudina; EFV: efavirenz; NVP: nevirapina; ETR: etravirina; TARV: terapia anti-retroviral.

\section{Criterios de inclusión y exclusión}

Se incluyó en el estudio a todos los pacientes que iniciaron o cambiaron tratamiento anti-retroviral en el período comprendido entre mayo y julio de 2011, previa autorización por parte del paciente a ingresar y cumplir con el programa de adherencia del establecimiento. Se excluyó del estudio pacientes que rechazaran asistencia semanal a controles.

\section{Resulltados}

\section{Características de la población estudiada}

En el estudio se incluyeron a 92 pacientes portadores del virus de inmunodeficiencia humana (VIH) que iniciaron o cambiaron TARV en el Hospital San Juan de Dios. El 77,2\% de los pacientes estudiados, fueron hombres y el grupo etario más prevalente al iniciar el seguimiento fue el de 30 a 39 años (34,8\%). La mayoría eran pacientes vírgenes a tratamiento. Respecto a la TARV iniciada, su mayor distribución correspondía al uso de dos inhibidores nucleosídicos de transcriptasa reversa (INTR) + un inhibidor de proteasa (IP), seguido del uso de dos INTR + un inhibidor no nucleósido de transcriptasa reversa (INNTR) (51,2 y 44,6\%, respectivamente) (Tabla 1$)$.

\section{Características de los eventos adversos pesquisados}

Se pesquisaron 76 eventos adversos en 69/92 pacientes durante el seguimiento (75\%); la reacción adversa de mayor incidencia fue la hiperbilirrubinemia indirecta asociada a atazanavir (ATV) en 15 de 18 pacientes, seguido de diarrea por lopinavir/ritonavir. (LPV/r) en 16 de los 29 pacientes expuestos al fármaco (Tabla 2).

Al evaluar la causalidad de los 76 eventos adversos detectados según el Algoritmo de Naranjo (Anexo 2) se obtuvo que $85 \%$ de ellas era "probable" y el restante $15 \%$ era "posible". Dentro de los resultados obtenidos ninguna reacción adversa calificó como dudosa ni definida.

Sobre el tiempo de exposición al fármaco para la manifestación del evento, éstas se detectaron en un período entre uno y tres meses de iniciada la TARV. Para el caso de diarrea por LPV/r, ésta comprendió un período entre siete y 30 días y una mediana de cinco días para su detección; en el caso de ictericia e hiperbilirrubinemia por ATV, ésta se presentó con una mediana de 14 días desde su introducción (Tabla 3).

Al clasificar estas alteraciones según la guía tabla entregada por el MINSAL, fue posible graduar 68 eventos, donde $34,1 \%$ tiene un mínimo grado de toxicidad (grado 1); $32,9 \%$ un grado 2 de toxicidad; $9,4 \%$ grado 3 y $3,5 \%$ alcanza el nivel más alto de toxicidad (grado 4) siendo en su totalidad debido a hiperbilirrubinemia indirecta por ATV. Cefaleas y náuseas no fueron graduadas al no contar con una escala que permita su clasificación en la guía clínica antes mencionada. 
Del total de pacientes que presentaron algún evento adverso, 22 de ellos $(31,8 \%)$ requirieron un cambio precoz de TARV (antes de siete días desde la indicación médica). Respecto a las causas de cambio de tratamiento, la más frecuente fue hematológica (anemia por AZT), seguido de la intolerancia gástrica (diarrea por LPV/r), con 45,5 y 27,3\%, respectivamente. No obstante estas alteraciones corresponden en su mayoría a toxicidades de grado 1 ó 2 (Figura 1).

\section{Manejo de eventos adversos}

El manejo de los eventos adversos observados fue muchas veces sintomático (por ej: en la aparición de exantema tratado con antihistamínicos y/o corticosteroides, diarrea por LPV/r con loperamida, intolerancia gástrica con inhibidores de la bomba de protones (omeprazol), antagonistas de histamina- $\mathrm{H}_{2}$ (ranitidina, famotidina) y/o con medidas no farmacológicas (por ej: ajuste de horario y restricción de alimentos ricos en grasas en mareos por $\mathrm{EFV}$, y en náuseas, modificación del horario de la toma, evitar alimentos calientes y/o muy condimentados, evitar aromas intensos, entre otras). Dado lo anterior, es de vital importancia informar al paciente de los posibles eventos adversos que podrían suceder al inicio del tratamiento. Si la reacción adversa no pone en peligro inmediato al paciente, se debe considerar manejo farmacológico y no farmacológico para controlar el o los síntomas o signos, manteniendo la misma terapia anti-retroviral. Si el paciente continúa con intolerancia a pesar de la optimización del manejo del efecto adverso, el medicamento considerado causante debe ser rápidamente reemplazado para no perjudicar la adherencia a la nueva TARV.

\section{Discusión}

De acuerdo a los resultados obtenidos del plan de seguimiento a pacientes con tratamiento anti-retroviral, se observa que la mayor distribución de pacientes se ubica en el rango de 30 a 39 años, seguido del rango de 40 a 49 años; esto concuerda con la realidad local según el último censo del departamento de epidemiología del Ministerio de Salud de 2007. A su vez existe una notable prevalencia de pacientes de sexo masculino $(77,2 \%)$, lo cual concuerda con el $82 \%$ informado en este mismo informe ${ }^{2}$.

En este estudio no se pudo realizar un análisis de género al no contar con un universo femenino estadísticamente comparativo en relación al número de género masculino.

De los 92 pacientes que iniciaron tratamiento, $75 \%$ de ellos presentó al menos un efecto adverso. Estudios sobre monitoreo de reacciones adversas notificadas espontáneamente, han descrito una incidencia de 47,7\% y una prevalencia variable de 12,3 a $39,7 \% 0^{3-4}$. En nuestro trabajo se consideraron y notificaron todos los efectos adversos esperables independientemente de la relevancia clínica de éstos.
Tabla 3. Período de detección del evento adverso notificado (n: 76). Estudio de reacciones adversas a terapia anti-retroviral. Hospital San Juan de Dios, Chile. 2011

\begin{tabular}{|c|c|c|c|}
\hline RAM & Causal & $\begin{array}{c}\text { Tiempo de exposición a } \\
\text { fármaco }\end{array}$ & $\begin{array}{l}\text { Mediana } \\
\text { (días) }\end{array}$ \\
\hline Intolerancia gastro-intestinal (diarrea) & $\mathrm{LPV} / \mathrm{r}$ & $\leq 1$ mes [7-30 días] & 5 \\
\hline Hiperbilirrubinemia indirecta & ATV/r & [7días-3 meses] & 14 \\
\hline Intolerancia (cefaleas, náuseas, vómitos) & \multirow{3}{*}{ AZT/3TC } & $\leq 1$ mes [7-30 días] & 3 \\
\hline Anemia & & $\leq 3$ meses [1-3 meses] & 45 \\
\hline Neuropatía periférica & & $\geq 3$ meses & 73 \\
\hline Alteración SNC & \multirow[b]{2}{*}{ EFV } & $<1$ mes [7-30 días] & 2,5 \\
\hline Rash cutáneo & & $<7$ días & 9 \\
\hline Rash cutáneo & NVP & $<40$ días & 35 \\
\hline Rash cutáneo & ETR & $<14$ días & 13 \\
\hline Hipercolesterolemia & \multirow{3}{*}{ TARV } & $\geq 3$ meses & 62 \\
\hline Hipertrigliceridemia & & $\geq 3$ meses & 62 \\
\hline Hepatotoxicidad & & $\geq 3$ meses & 53 \\
\hline
\end{tabular}

RAM: reacción adversa a medicamentos; LPV: lopinavir/ritonavir; ATV/r: atazanavir/ritonavir; AZT/3TC: zidovudina/lamivudina; EFV: efavirenz; NVP: nevirapina; ETR: etravirina; TARV: terapia antiretroviral.

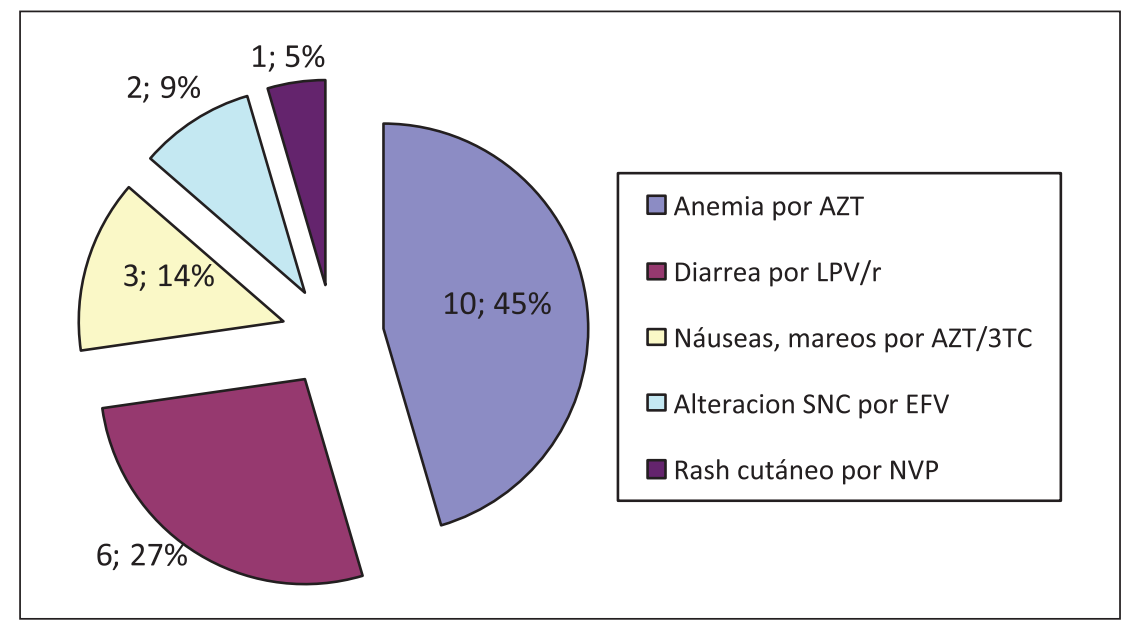

Figura 1. Cambios de TARV y su causal (n: 22). AZT: zidovudina; LPV/r: lopinavir/ritonavir; AZT/3TC: ziduvudina/lamivudina; EFV: efavirenz; NVP: nevirapina.

Dentro de los eventos adversos más prevalentes destaca la diarrea por LPV/r seguido de la hiperbilirrubinemia indirecta por ATV. Sobre esta última, un estudio de asignación aleatoria, abierto y multicéntrico, observó que menos de $1 \%$ de los pacientes suspende tratamiento como consecuencia de la hiperbilirrubinemia, ictericia o ictericia escleral antes de la semana 48; sin observarse diferencias en la calidad de vida de los pacientes (con o sin hiperbilirrubinemia). A lo largo del estudio, se observó además una menor tolerancia a LPV/r al provocar mayor diarrea y náuseas que su comparador $(\mathrm{ATV} / \mathrm{r})^{5-7}$.

Es importante resaltar que en este estudio no se 
registraron reacciones adversas como lipodistrofia y/o lipoatrofia porque éstas suelen presentarse a largo plazo, con un tiempo de administración de TARV mayor a un año, como se han demostrado en otros estudios ${ }^{8-10}$. Esto podría considerarse como una limitación del estudio; sin embargo, el objetivo del seguimiento es la evaluación de los eventos adversos precoces del tratamiento antiretroviral.

El motivo más frecuente de cambio de tratamiento en este estudio fue la anemia 13,2\% (n: 10) del total de eventos adversos pesquisados. Otros estudios han publicado una incidencia de anemia asociada a AZT que oscilan entre 10 y $35 \%$, sin dejar de lado que existen factores inmunológicos, virológicos y características propias de los pacientes, que inciden en la aparición de estas alteraciones ${ }^{11-14}$. Por otro lado, la Cohorte Chilena de SIDA ha informado como causa de cambio o suspensión del tratamiento por toxicidad, las anemias constituyendo el $9,9 \%{ }^{15}$. Esta alta incidencia de anemia puede asociarse al hecho que en Chile el uso del análogo nucleosídico AZT, es considerado uno de los anti-retrovirales a elección en pacientes vírgenes a TARV para constituir la columna vertebral del tratamiento anti-retroviral. Una estrategia para disminuir la incidencia de de este evento podría ser la sustitución por análogos con menor riesgo de toxicidad hematológica como abacavir o tenofovir.

Las causales de cambios de TARV corresponden a eventos adversos a corto plazo. No obstante, el seguimiento continuo a través del ciclo de vida de los paciente, donde se considere la evaluación del impacto metabólico, es fundamental para la detección temprana de toxicidades a largo plazo tales como alteraciones de sistema nervioso periférico, alteraciones metabólicas con redistribución de la grasa corporal y dislipidemias las cuales pueden repercutir de forma negativa en la adhesión al tratamiento, eficacia de la terapia y desarrollo de resistencia a los fármacos utilizados ${ }^{14-18}$.

\section{Conclusiones}

Durante los primeros meses de tratamiento es esperable un alto porcentaje de RAM (75\% en nuestro estudio). El conocimiento de la real incidencia de efectos adversos puede ayudar a los equipos tratantes a un manejo adecuado y contención de éstos en el paciente, con el fin de no realizar un cambio anticipado de TARV.

En nuestro centro fue necesario realizar un cambio de TARV en $31,8 \%$ de los pacientes que experimentaron una RAM. La actitud y conocimiento frente al desarrollo de eventos adversos a corto plazo constituye la base del manejo de la toxicidad farmacológico a ARV. Por un lado, la interrupción de un tratamiento suele ser necesario en el manejo de RAM que aparecen precozmente y suponen un peligro para la vida del paciente, como por ejemplo: un exantema intenso a nevirapina, anemia grave por AZT. Sin embargo, la mayoría de los efectos adversos pueden ser corregidos en forma parcial o total mediante intervenciones farmacológicas y no farmacológicas.

El mayor porcentaje de los cambios realizados por RAM fueron de grado 1 y 2, correspondientes a pacientes vírgenes de tratamiento que iniciaron TARV; las alteraciones grado 3 fueron aisladas, y aquellas grado 4 correspondieron en su totalidad a hiperbilirrubinemias por ATV, lo cual clínica y estéticamente no fue un inconveniente para nuestros pacientes. Es importante destacar que las alteraciones grado $4(n=3)$ sólo se expresaron con leve ictericia escleral y en los exámenes de laboratorio (con aumento de bilirrubina indirecta), en rangos de 5,52-5,75 y 5,75 mg/dL respectivamente. (Rango referencia: 0,05-1,1mg/dL). Junto con lo anterior un factor que podría considerarse como protector frente a la presencia de ictericia es un tono de piel mestizo o trigueño, en comparación a uno caucásico.

La integración del químico farmacéutico al equipo profesional que atiende pacientes con infección por VIH/ SIDA es fundamental y debe tener un rol activo en el control de las personas infectadas, colaborando en la detección temprana de los eventos adversos, su contención, manejo y evitando así un cambio precoz de tratamiento. La implementación de un programa de adherencia, protocolos o guías de manejo de RAM a anti-retrovirales, y el uso de herramientas para la graduación de su toxicidad (Anexo 1), puede ser de ayuda en la contención de estas molestias. Se debe concientizar al paciente en el inicio o cambio de su tratamiento sobre los eventos adversos esperados y pasajeros de los anti-retrovirales; y frente a la pesquisa de dichos eventos, se debe aplicar criterio clínico para la evaluación de dicha graduación de toxicidad. (Por ejemplo: en hiperbilirrubinemia por ATV, se debe evaluar el grado de impacto en la calidad de vida del paciente más que el valor del análisis de laboratorio).

\section{Resumen}

Introducción: En la Cohorte Chilena de SIDA, 15,9\% de los pacientes requiere cambio de su primer esquema de TARV por toxicidad, siendo además la causa de $47 \%$ de las suspensiones de tratamiento ocurrida en los primeros meses de terapia. Objetivo: Analizar la incidencia y causalidad de las reacciones adversas a medicamentos en pacientes que inician o cambian terapia anti-retroviral. Material y Métodos: Se realizó un seguimiento para la detección de reacciones adversas a medicamentos a 92 pacientes que iniciaron o cambiaron tratamiento anti-retroviral en un período comprendido entre mayo y julio del 2011. Resultados: 75\% (n: 69) de los pacientes presentó al menos un evento adverso. De las 76 reacciones adversas a medicamentos detectadas, las más frecuentes fueron: 
diarrea $(21,1 \%$; n: 16) e hiperbilirrubinemia $(19,7 \% \mathrm{n}$ : 15). El $85,9 \%$ de las notificaciones se calificó según su gravedad; obtuvo una toxicidad grado 1 en $34,1 \% ; 32,9 \%$ grado $2 ; 9,4 \%$ grado 3 y $3,5 \%$ grado 4 . Sólo 22 pacientes $(31,8 \%)$ requirieron un cambio de tratamiento, las causas más frecuentes de cambio fueron: anemia por AZT (45,4\%; n: 10) y diarrea asociada al uso de LPV/r (27,3\%; n: 6). Conclusión: El conocimiento de la real incidencia de estas reacciones adversas a medicamentos puede ayudar a los equipos tratantes para un adecuado control y manejo de éstos en el paciente, con el fin de evitar un cambio anticipado de TARV. Es fundamental conformar un equipo multidisciplinario que incluya al químico farmacéutico para una adecuada farmacovigilancia.

Anexo 1. Graduación de la toxicidad según la Guía Clínica Ministerio de Salud de Chile para infección por VIH/SIDA del año 2009

\begin{tabular}{|c|c|c|c|c|}
\hline Graduación de las principales toxicidades & Grado 1 & Grado 2 & Grado 3 & Grado 4 \\
\hline Hematocrito (\%) & $28,5-31,4$ & $24-28,4$ & $19,5-23,9$ & $<19,5$ \\
\hline Hemoglobina (g/100 ml) & $9,5-11$ & $8,0-9,4$ & $6,5-7,9$ & $<6,5$ \\
\hline Leucocitos $\left(\mathrm{mm}^{3}\right)$ & $2.500-3.999$ & $1.000-2.499$ & $800-999$ & $<800$ \\
\hline Recuento neutrófilos $\left(/ \mathrm{mm}^{3}\right)$ & $1.000-1.499$ & $750-999$ & $500-749$ & $<500$ \\
\hline Recuento plaquetas $\left(\mathrm{mm}^{3}\right)$ & $75.000-99.000$ & $50.000-74.999$ & $20.000-49.999$ & $<20.000$ o petequias \\
\hline Hiperglicemia (mg \%) & $116-160$ & $161-250$ & $251-500$ & > 500 o cetoacidosis \\
\hline Hipertrigliceridemia (mg \%) & $250-400$ & $401-750$ & $751-1250$ & $>1250$ \\
\hline Acidosis metabólica (HCO3: mEq/lt) & $19-21$ & $15-18$ & $10-14$ & $<10$ \\
\hline Creatininemia (x límite máx normal) & $1,1-1, .5$ & $1,6-3,0$ & $3,1-6,0$ & > 6 o diálisis \\
\hline Bilirrubinemia (x límite máx normal) & $1,1-1,5$ & $1,6-2,5$ & $2,6-5,0$ & $>5$ \\
\hline GOT (x límite máx normal) & $1,25-2,5$ & $2,6-5,0$ & $5,1-10,0$ & $>10$ \\
\hline GPT (x límite máx normal) & $1,25-2,5$ & $2,6-5,0$ & $5,1-10,0$ & $>10$ \\
\hline GGT (x límite máx normal) & $1,25-2,5$ & $2,6-5,0$ & $5,1-10,0$ & $>10$ \\
\hline Fosfatasas alcalinas (x límite máx n) & $1,25-2,5$ & $2,6-5,0$ & $5,1-10,0$ & $>10$ \\
\hline Lipasemia (x límite máx normal) & $1,1-1,39$ & $1,4-2,09$ & $2,1-5,0$ & $>5$ o pancreatitis \\
\hline Amilasemia (x límite máx normal) & $1,1-1,39$ & $1,4-2,09$ & $2,1-5,0$ & $>5$ o pancreatitis \\
\hline Vómitos & Aislados & Frecuentes & $\begin{array}{l}\text { Hipotensión arterial o } \\
\text { hidratación EV }\end{array}$ & $\begin{array}{l}\text { Hipotensión arterial grave } \\
\text { y/o hospitalización }\end{array}$ \\
\hline Diarrea & 3-4 x día & 5-7 y/o nocturna & $\begin{array}{l}>7 \text {, hipotensión arterial, } \\
\text { hidratación EV }\end{array}$ & $\begin{array}{l}\text { Hipotensión arterial grave } \\
\text { y/o hospitalización }\end{array}$ \\
\hline Litiasis renal & \multicolumn{4}{|c|}{ Grado 4: Hematuria grave y/o insuficiencia renal obstructiva } \\
\hline Polineuropatía & Leve & Requiere tratamiento & Interfiere con el sueño & Limita la marcha \\
\hline Alteración del SNC & Ansiedad o depresión leve & Moderada & Grave, requiere asistencia & $\begin{array}{l}\text { Psicosis aguda y/o hospita- } \\
\text { lización }\end{array}$ \\
\hline Alergia & Rash leve y/o prurito & $\begin{array}{l}\text { Máculas o máculo-pápulas } \\
\text { difusas }\end{array}$ & Generalizadas & $\begin{array}{l}\text { Anafilaxis, Stevens Johnson } \\
\text { o exfoliación }\end{array}$ \\
\hline Hipersensibilidad a abacavir & \multicolumn{4}{|c|}{ Grado 4: Rash con fiebre y/o síntomas digestivos o respiratorios } \\
\hline
\end{tabular}

\section{Anexo 2. Algoritmo de Naranjo}

1. ¿Existen notificaciones concluyentes sobre esta reacción?

2. ¿Se produjo la reacción adversa después de administrar el fármaco sospechoso?

3. ¿Mejoró la reacción adversa tras suspender la administración del fármaco o tras administrar un antagonista específico?

4. ¿Reapareció la reacción adversa tras la re-administración del fármaco?

5. ¿Existen causas alternativas (diferentes del fármaco) que podrían haber causado la reacción por sí misma?

6. ¿Reapareció la reacción adversa tras administrar un placebo?

7. ¿Se detectó el fármaco en la sangre (o en otros fluidos) en concentraciones tóxicas?

8. ¿Fue la reacción más intensa al aumentar la dosis o menos intensa al disminuirla?

9. ¿Tuvo el paciente alguna reacción similar causada por el mismo fármaco u otro semejante en cualquier exposición anterior?

10. ¿Se confirmó el acontecimiento adverso por cualquier tipo de evidencia objetiva?

Tipo de RAM:

\begin{tabular}{|c|c|c|c|c|}
\hline Sí & No & No sabe & & \\
\hline 1 & 0 & 0 & & \\
\hline 2 & -1 & 0 & & \\
\hline 1 & 0 & 0 & & \\
\hline 2 & -1 & 0 & & \\
\hline-1 & 2 & 0 & & \\
\hline-1 & 1 & 0 & & \\
\hline 1 & 0 & 0 & & \\
\hline 1 & 0 & 0 & & \\
\hline \multirow[t]{2}{*}{1} & 0 & 0 & Tipo & Puntuación \\
\hline & & & Definida & 9 o más puntos \\
\hline \multirow[t]{3}{*}{1} & 0 & 0 & Probable & 5-8 puntos \\
\hline & & & Posible & 1-4 puntos \\
\hline & & & Dudosa & 0 ó menos puntos \\
\hline
\end{tabular}

ref: apps.who.int/medicinedocs/es/d/Js8121s/7.7.html 


\section{Referencias bibliográficas}

1.- Wolff M J, Cortés C P, Shepherd B E, Beltrán C J. Chilean AIDS Cohort Study Group. Long-term outcomes of a national expanded access program to antiretroviral therapy: the Chilean AIDS cohort. J Acquir Immune Defic Syndr 2010; 55 (3): 368-74.

2.- MINSAL. "Evolución del VIH-SIDA V Región, 1987-2006”, Departamento de Epidemiología, DIPLAS 2008, agosto.

3.- Modayil R R, Harugeri A, Parthasarathi G, Ramesh M, Prasad R, Naik V, et al. Adverse drug reactions to antiretroviral therapy (ART): an experience of spontaneous reporting and intensive monitoring from ART centre in India. Pharmacoepidemiol Drug Saf 2010; 19 (3): 247-55.

4.- Anwikar S R, Bandekar M S, Smrati B, Pazare A P, Tatke P A, Kshirsagar N A. HAART induced adverse drug reactions: A retrospective analysis at a tertiary referral health care center in India. Int J Risk Saf Med 2011; 23 (3): 163-9.

5.- McDonald C, Uy J, Hu W, Wirtz V, Juethner S, Butcher D, et al. Clinical significance of hyperbilirubinemia among HIV-1-infected patients treated with atazanavir/ritonavir through 96 weeks in the CASTLE study. AIDS Patient Care STDS 2012; 26 (5): 259-64.

6.- Smith D E, Jeganathan S, Ray J. Atazanavir plasma concentrations vary significantly between patients and correlate with increased serum bilirubin concentrations. HIV Clin Trials 2007, 7: 34-8.

7.- Rodríguez-Nóvoa S, Martín-Carbonero L, Barreiro P, González-Pardo G, Jiménez-Nácher I, González-Lahoz J, et al. Genetic factors influencing atazanavir plasma concentrations and the risk of severe hyperbilirubinemia. AIDS 2007; 21: 41-6.

8.- Pujari S N, Dravid A, Naik E, Bhagat S,
Tash K, Nadler J P, et al. Lipodystrophy and dyslipidemia among patients taking first-line, World Health Organization-recommended highly active antiretroviral therapy regimens in Western India. J Acquir Immune Defic Syndr 2005; 39 (2): 199-202.

9.- Friis-Møller N, Weber R, Reiss P, Thiebaut R, Kirk O, d'Arminio Monforte A, et al. Cardiovascular disease risk factors in HIV patients-association with antiretroviral therapy. Results from the DAD study. AIDS 2003; 17 (8): 1179-93

10.- Martínez E, Mocroft A, García-Viejo M A, Pérez-Cuevas J B, Blanco J L, Mallolas J, et al. Risk of lipodystrophy in HIV-1 infected patients treated with protease inhibitors: a prospective cohort study. Lancet 2001; 357: 592-8.

11.- Rajeash R H, Vidyasagar S, Varma D M, Mohiuddin S, Noorunnisa. Evaluation of incidence of zidovudine induced anemia in Indian human immunodeficiency virus positive patients in comparison with stavudine based highly active antiretroviral therapy. Int J Risk Saf Med 2011; 23 (3): 171-80

12.- Pádua C A, César C C, Bonolo P F, Acurcio F A, Guimarães M D. Self-reported adverse reactions among patients initiating antiretroviral therapy in Brazil. Braz J Infect Dis 2007; 11 (1): 20-6.

13.- Pádua C A, César C C, Bonolo P F, Acurcio F A, Guimarães M D. High incidence of adverse reactions to initial antiretroviral therapy in Brazil. Braz J Med Biol Res 2006; 39 (4): 495 505 .

14.- Astuvilca J, Arce-Villavicencio Y, Sotelo R, Quispe J, Guillén R, Peralta L, et al. Incidencia $\mathrm{y}$ factores asociados con las reacciones adversas del tratamiento antirretroviral inicial en pacientes con VIH. Rev. Perú. Med Exp Salud Pública 2007; 24 (3): 218-24. ISSN 1726-4634.

15.- Ministerio de Salud. 2007. Evaluación de impacto del programa de acceso expandido a tratamiento antirretroviral. Comisión Nacional de SIDA, Santiago; Chile.

16.- Cantwell-McNelis K, James C W. Role of clinical pharmacists in outpatient HIV clinics. Am J Health-Syst Pharm 2002; 59: 447-52.

17.- Max B, Sherer R. Management of adverse effects of antiretroviral therapy and medication adherence. Clin Infect Dis 2000; 30 (2): S96-116.

18.- Hawkins T. Understanding and managing the adverse effects of antiretroviral therapy. Antivir Res 2010; 85: 201-9.

19.- Sulkowski M S, Thomas D L, Chaisson R E, Moore R D. Hepatotoxicity associated with antiretroviral therapy in adults infected with human immunodeficiency virus and the role of hepatitis $\mathrm{C}$ or $\mathrm{B}$ virus infection. JAMA 2000; 283 (1): 74-80.

20.- Keswani S C, Pardo C A, Cherry C L, Hoke A, McArthur J C. HIV-associated sensory neuropathies. AIDS 2002; 16: 2105-17.

21.- Domingo P, Lozano F. Manejo de la toxicidad por fármacos antiretrovirales. Enferm Infecc Microbiol Clin 2011; 29 (7): 535-44.

22.- Clifford D B, Evans S, Yang Y, Acosta E P, Goodkin K, Tashima K, et al. Impact of efavirenz on neuropsychological performance and symptoms in HIV-infected individuals. Ann Intern Med 2005; 143: 714-21.

23.- Schiller D S. Identification, management and prevention of adverse effects associated with highly active antiretroviral therapy. Am J Health Syst Pharm 2004; 61: 2507-22.

24.- Antón P, Soriano V, Jiménez-Nácher I, Rodríguez-Rosado R, Dona M C, Barreiro P M, et al. Incidence of rash and discontinuation of nevirapine using two different escalating initial doses. AIDS 1999; 13: 524-5.

25.- Soriano V, Puoti M, García-Gascó P, Rockstroh J K, Benhamou Y, Barreiro P, et al. Antiretroviral drugs and liver injury. AIDS 2008; 22: 1-13. 